Books, videos, CD-ROMs, DVDs and any other relavent items submitted for a review in the $B D J$ should be addressed to: Kate Maynard, Assistant Editor, British Dental Journal, NPG, 4-6 Crinan Street, London, N1 9XW

\title{
Manual of minor oral surgery for the general dentist
}

\begin{abstract}
K. R. Koerner
UK: Blackwell Munksgaard

price $€ 49.50$, pp 336

ISBN 0813805597

This is an American text with the stated aim of providing the general dental practitioner with a practical guide to oral surgery procedures that are performed daily in general dental practice. The editor is a general dental practitioner working in private practice limited to oral surgery. He is an international lecturer and has been teaching clinical courses on oral surgery to other dentists in the United States since 1981. He has brought together nine other contributors of varying seniority and backgrounds from the United States, all sharing many pearls of wisdom gleaned from years of experience and training, to increase readers' confidence and competence.
\end{abstract}

The ability to carry out minor oral surgery procedures can be a daunting task for the busy general dental practitioner and is something which is under taught at undergraduate level. This often results in referrals to secondary or specialist centres. This book provides a good starting point for someone with an interest in oral surgery trying to increase the amount of surgical work they carry out. It also presents more advanced procedures for those experienced dentists wishing to develop professionally.

The book is divided into 11 chapters, each of which is very clear, concise and well-referenced throughout. They cover a wide range of clinical techniques from surgical extractions, the removal of impacted wisdom teeth, pre-prosthetic surgery, endodontic periradicular microsurgery, surgical crown lengthening and biopsy. Each clinical procedure described is richly illustrated with clinical photographs, diagrams and radiographs which help the reader to visualise the treatment described.
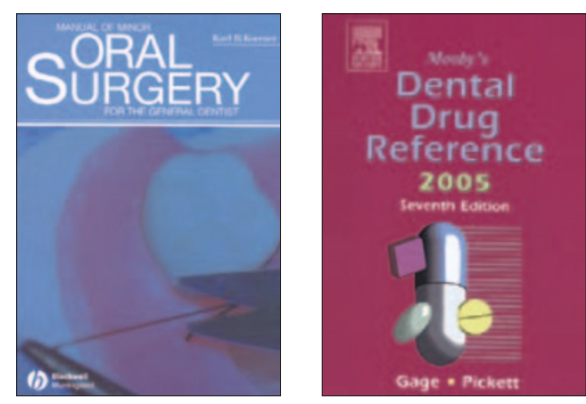

However, some techniques are not covered in enough detail to allow the reader to carry out the clinical procedure and surgical periodontal treatment has been completely omitted.

Supportive topics included are patient evaluation and case selection, management of post-operative complications and the use of anxiolysis for oral surgery. The book also touches upon medico-legal recommendations and safety requirements needed in today's increasingly litigious society. The final chapter focuses on organising and conducting trips to Third World countries where the skills developed can be used. It aims to encourage more altruistic humanitarian service.

In summary, this is an excellent book supported by high-quality illustrations and appropriate text layout containing much sensible and practical advice. It would prove a valuable reference to all general dental practitioners who have an interest in oral surgery.

V. Gohel

\section{Mosby's dental drug reference}

\section{T. W. Gage, F. A. Pickett \\ UK: Elsevier \\ price $€ 23.99$, pp 1,008 \\ ISBN 0323040810}

With an ageing population dentists will be called upon more frequently to treat medically compromised patients. These patients could be taking several medications at any given time. This socalled polypharmacy can affect treatment planning and it is essential clinicians are aware of the possible dental implications. Currently the British National Formulary (BNF) and the Dental Practitioner's Formulary (DPF) are available to both dentists and dental care professionals (DCPs) as an aid and reference of prescription medications. This book aims to be an easy to use, quick and concise source which can be used at the chairside.

This book has a range of contributors from dental, medical and surgical backgrounds. The first section separates drugs by pharmacological action. This is a very useful feature especially when faced with a patient who can recall the condition they suffer with but not their medication. This provides a quick reference and allows the clinician to search further information effectively. Drugs are listed alphabetically and each is described in a set format. This consists of the generic name and its pronunciation, trade names, drug classification, whether it is a controlled drug, mode of action and uses, doses and routes of administration, sideeffects, contraindications, precautions, pharmacokinetics, and drug interactions/ considerations related to dentistry. This is really the main selling point of this book. It is an excellent feature which is easy to follow and provides relevant information which could affect dental treatment planning. The enclosed CD-ROM contains a total of 30 useful intraoral/extraoral colour photographs of common oral manifestations of specific drugs. Each figure has an accompanying legend and is cross-referenced to the corresponding page in the textbook. In total 950 patient information sheets on specific medications which can be printed and given to patients is also included on the CD-ROM. This feature can be used in any clinical environment and provides the patient with relevant information such as side-effects, dosages etc. The appendices cover a range of topics which include drugs that cause xerostomia and herbal drugs. Bacterial endocarditis prophylactic regimens for dental procedures are described but are cited from the American Heart Association guidelines (1997).

Generally this textbook satisfies its aims and will appeal to GDPs, hospital clinicians and DCPs. UK clinicians should use this textbook as an adjunctive to the current BNF and DPF rather than a primary source and follow the endocarditis guidelines as recommended by the British Society for Antimicrobial Chemotherapy (2006).

J. Seehra 